PROCEEDINGS OF THE

AMERICAN MATHEMATICAL SOCIETY

Volume 126, Number 12, December 1998, Pages 3467-3473

S 0002-9939(98)04379-2

\title{
COMPLETELY LEXSEGMENT IDEALS
}

\author{
EMANUELA DE NEGRI AND JÜRGEN HERZOG
}

(Communicated by Wolmer V. Vasconcelos)

\begin{abstract}
In this paper we study ideals which are generated by lexsegments of monomials. In contrast to initial lexsegments, the shadow of an arbitrary lexsegment is in general not again a lexsegment. An ideal generated by a lexsegment is called completely lexsegment, if all iterated shadows of the set of generators are lexsegments. We characterize all completely lexsegment ideals and describe cases in which they have a linear resolution. We also prove a persistence theorem which states that all iterated shadows of a lexsegment are again lexsegments if the first shadow has this property.
\end{abstract}

\section{INTRODUCTION}

In the theory of extremal combinatorics as well as in the theory of Hilbert functions lexsegment ideals play an important role. Macaulay's fundamental theorem on Hilbert functions can be stated in the following form: Let $k$ be a field, and $I$ a graded ideal in a polynomial ring $k\left[x_{1}, \ldots, x_{n}\right]$. Then there is a unique lexsegment ideal, denoted $I^{l e x}$, which has the same Hilbert function as $I$. In the usual terminology, a lexsegment ideal is a monomial ideal which in each degree is spanned by an initial lexsegment of monomials. Recall that an initial lexsegment in degree $d$ is a set of monomials of the form $\left\{w \in M_{d}: w \geq u\right\}$ where $M_{d}$ denotes the set of all monomials of degree $d, u \in M_{d}$, and where $>$ is the lexicographic term order with $x_{1}>x_{2}>\ldots>x_{n}$. We denote the above set by $L^{i}(u)$. The exponent $i$ indicates that the set considered is an initial lexsegment. One may as well consider final lexsegments, that is, sets $L^{f}(u)=\left\{w \in M_{d}: w \leq u\right\}$, or even more general arbitrary lexsegments $L(u, v)=\left\{w \in M_{d}: u \geq w \geq v\right\}$. Arbitrary lexsegments have first been studied by Hulett and Martin [HM].

One important property of initial lexsegments, which is crucial in the proof of Macaulay's theorem, is in general no longer satisfied by arbitrary lexsegments. It is the property that the shadow of a lexsegment is again a lexsegment. The shadow of a set $S$ of monomials is the set $\operatorname{Shad}(S)=\left\{v x_{i}: v \in S, i=1, \ldots, n\right\}$. We define the $i$-th shadow recursively by $\operatorname{Shad}^{i}(S)=\operatorname{Shad}\left(\operatorname{Shad}^{i-1}(S)\right)$. The simplest examples of lexsegments whose shadow in general is not a lexsegment are those lexsegments which consist of just one element.

Hulett and Martin call a lexsegment $L$ completely lexsegment if all the iterated shadows of $L$ are again lexsegments, that is, if for each $i$ the set $\operatorname{Shad}^{i}(L)$ is a lexsegment. In their paper they give a sufficient condition for a lexsegment to be

Received by the editors February 7, 1997 and, in revised form, March 5, 1997.

1991 Mathematics Subject Classification. Primary 13C99, 13D02.

(C) 1998 American Mathematical Society 
completely lexsegment. The main purpose of this paper is to give a full characterization of the lexsegments which are completely lexsegment. Our result is described in Theorem 2.3 .

In the first section of the paper we develop some of the techniques needed in the proofs of Section 2, and also show the following persistence theorem for lexsegments: A lexsegment whose shadow is again lexsegment is completely lexsegment, that is, if the first shadow is a lexsegment, then all iterated shadows are lexsegments as well.

All our proofs in this paper are based on the fact that an arbitrary lexsegment is the intersection of an initial and a final lexsegment. We also use the fact that, according to a result $[\mathrm{AH}]$ of Aramova and the second author of this paper, the Koszul cycles of the initial and final lexsegment ideals are known. Indeed, both ideals are stable - the initial lexsegment ideals in the usual sense, that is, in the sense of Eliahou and Kervaire, the final lexsegment ideals with the order of the variables reversed. These observations are used in particular in the final section where we study the resolution of ideals generated by arbitrary lexsegments. It is well-known, and follows easily from the Eliahou-Kervaire resolution [EK], that the initial lexsegment ideals have linear resolutions. Again this property fails in general for arbitrary lexsegment ideals. We show however that a large class of completely lexsegment ideals do have a linear resolution. A similar result was proved by Hulett and Martin in a more special case.

In concluding we want to remark that the subring of the polynomial ring which is generated by a lexsegment is always a Koszul algebra, regardless whether or not the lexsegment is completely lexsegment. This was shown by De Negri in [D].

The authors would like to thank Annetta Aramova for a careful reading of the first version of this paper and for providing an example showing that Theorem 3.1 in its original form was not quite correct.

\section{A PeRsistence theorem For LeXSEGMENT ideALS}

Throughout this paper $R=k\left[x_{1}, \ldots, x_{n}\right]$ is the polynomial ring over a field $k$, and $<$ denotes the deglex order on the monomials of $R$. Let $u=x_{1}^{a_{1}} \ldots x_{n}^{a_{n}}$ and $v=x_{1}^{b_{1}} \ldots x_{n}^{b_{n}}$ be two monomials. Then $u<v$, if either $\operatorname{deg} u<\operatorname{deg} v$, or else $\operatorname{deg} u=\operatorname{deg} v$ and there exists an integer $i, 1 \leq i \leq n$, such that $a_{j}=b_{j}$ for $j=1 \ldots, i-1$ and $a_{i}<b_{i}$. Thus, for example, the set of quadratic monomials in three variables in deglex order is

$$
x_{1}^{2}>x_{1} x_{2}>x_{1} x_{3}>x_{2}^{2}>x_{2} x_{3}>x_{3}^{2} .
$$

We call an ideal of $R$ a lexsegment ideal if it is generated by an (arbitrary) lexsegment. A homogeneous component of a graded ideal will be called lexsegment if it is spanned as $k$-vector space by a lexsegment. Moreover, we call a graded ideal completely lexsegment, if all components of $I$ are lexsegment.

This section is devoted to the proof of

Theorem 1.1 (Persistence). Let I be a lexsegment ideal generated in degree d, and suppose that $I_{d+1}$ is lexsegment. Then $I$ is completely lexsegment.

For the proof of the theorem we shall need two facts.

Lemma 1.2. Let $u \geq v$ be two monomials in $M_{d}, I=(L(u, v))$ and $K=\left(L^{f}(u)\right)$. If $I_{d+1}$ is lexsegment, then $K_{d+1}$ is lexsegment. 
Proof. Since $\operatorname{Shad}\left(L^{i}(u)\right)=L^{i}\left(u x_{n}\right)$ and $\operatorname{Shad}\left(M_{d}\right)=M_{d+1}$, it follows that

$$
L^{f}\left(u x_{n}\right)=M_{d+1} \backslash\left\{w \in M_{d+1}: w>u x_{n}\right\} \subset \operatorname{Shad}\left(L\left(u, x_{n}^{d}\right)\right)=\operatorname{Shad}\left(L^{f}(u)\right) .
$$

By hypothesis, $\operatorname{Shad}(L(u, v))=L\left(u x_{1}, v x_{n}\right)$; therefore we have

$$
L^{f}\left(u x_{1}\right)=L\left(u x_{1}, v x_{n}\right) \cup L^{f}\left(u x_{n}\right) \subset \operatorname{Shad}\left(L^{f}(u)\right) .
$$

This implies that $L^{f}\left(u x_{1}\right)=\operatorname{Shad}\left(L^{f}(u)\right)$, since the other inclusion is trivially true. In particular, the shadow of $L^{f}(u)$ is a lexsegment, as desired.

Here and in later sections of the paper we shall have to consider the vector spaces $\operatorname{Tor}_{i}^{R}(k, R / I)$ for graded ideals $I$ of $R$. For simplicity we denote these vector spaces by $T_{i}(I)$. Note that the $T_{i}(I)$ inherit a natural grading from $I$. For each $j$ the $k$ dimension of $T_{i}(I)_{j}$ is the number of $j$-shifts in the $i$-th step of the graded minimal free $R$-resolution of $R / I$.

Lemma 1.3. Let $u \geq v$ be two monomials in $M_{d}, J=\left(L^{i}(v)\right)$ and $K=\left(L^{f}(u)\right)$. Then $I=J \cap K$ is generated in degree $d$ and $d+1$.

Proof. The ideal $J$ is stable in the sense of Eliahou and Kervaire, that is, it satisfies the following property: if $u \in J$ is a monomial and $x_{i}$ divides $u$, then $x_{j}\left(u / x_{i}\right) \in J$ for all $j \leq i$. It follows from the Eliahou-Kervaire resolution [EK] that stable ideals which are generated in one degree have a linear resolution. Therefore, $T_{i}(J)_{j}=0$ for all $i>0$ and $j \neq i+d-1$. Next we note that the ideal $K$ is also stable, but with the order of the variables reversed. In other words, if $u \in K$ is a monomial in $K$ and $x_{i}$ divides $u$, then $x_{j}\left(u / x_{i}\right) \in K$ for all $j \geq i$. Therefore, we may again apply the Eliahou-Kervaire result, and conclude that $K$ has a linear resolution, too.

Now consider the natural exact sequence

$$
0 \rightarrow R / I \rightarrow R / J \oplus R / K \rightarrow R / L \rightarrow 0,
$$

where $L=J+K$ is the ideal spanned by all monomials of degree $d$. Note that $L$ again has a linear resolution.

The above exact sequence gives rise to the following long exact sequence:

$$
\ldots \rightarrow T_{i+1}(L) \rightarrow T_{i}(I) \rightarrow T_{i}(J) \oplus T_{i}(K) \rightarrow T_{i}(L) \rightarrow \ldots
$$

Since $T_{i}(J)_{j}=T_{i}(K)_{j}=0$ for $i>0$ and $j \neq i+d-1$, and since $T_{i+1}(L)_{j}=0$ for $i>0$ and $j \neq i+d$, we conclude from the exact sequence (1) that $T_{i}(I)_{j}=0$ for $i>0$ and $j \neq i+d-1, i+d$. In particular, we have $T_{1}(I)_{j}=0$ for $j \neq d, d+1$. Hence the assertion follows.

Remark 1.4. The proof of 1.3 not only shows that $I$ is generated in degree $d$ and $d+1$, but also that the resolution of $I$ has shift only in two strands, the linear strand and the next higher one.

Now we are ready for the proof of the persistence theorem.

Proof of Theorem 1.1. Let $I=(L(u, v))$; we will show that $I=J \cap K$ where $J=\left(L^{i}(v)\right)$ and $K=\left(L^{f}(u)\right)$, and that $K$ is completely lexsegment. Since an initial lexsegment ideal is always completely lexsegment, this will then imply that $I$ is completely lexsegment.

Since $I_{d+1}$ is lexsegment it follows from 1.2 that $K_{d+1}$ is lexsegment. Therefore $(J \cap K)_{d+1}$ is lexsegment, and $(J \cap K)_{d+1}=I_{d+1}$, since both vector spaces are spanned by $L\left(u x_{1}, v x_{n}\right)$. Therefore, 1.3 implies that $I=J \cap K$. It remains to show 
that $K$ is a completely lexsegment ideal. But since $K_{d+1}$ is lexsegment, this follows from Proposition 2.2 in the next section.

\section{A CHARACTERIZATION OF COMPLETELY LEXSEGMENT IDEALS}

In this section we will give a characterization of the completely lexsegment ideals. In a first step we characterize the ideals generated by a final lexsegment which are completely lexsegment.

The following notation will be used: let $u$ be a monomial. We write $x_{i} \mid u$ to indicate that $x_{i}$ divides $u$, and set $m(u)=\min \left\{i: x_{i} \mid u\right\}$ and $M(u)=\max \left\{i: x_{i} \mid u\right\}$. Furthermore we set $u^{\prime}=u / x_{M(u)}$ and $u^{\prime \prime}=u / x_{m(u)}$.

The following result which will be needed in the proof of 2.2 is due to Hulett and Martin [HM, Theorem 3.1].

Lemma 2.1. Let $u, v \in M_{d}, u \geq v$, be such that $x_{1} \mid u$, and $u / x_{1} \geq v / x_{M(v)}$. Then $\operatorname{Shad}(L(u, v))$ is a lexsegment, too.

The equivalence of (b) and (c) in the following Proposition was first shown by Deery $[\mathrm{De}]$.

Proposition 2.2. Let $u \in M_{d}$, and let $K \subset R$ be the final lexsegment ideal generated by $L^{f}(u)$. Then the following conditions are equivalent:

(a) $K$ is completely lexsegment;

(b) $K_{d+1}$ is a lexsegment;

(c) $u \geq x_{2}^{d}$.

Proof. It suffices to prove the equivalence of (b) and (c). Indeed, if (c) is satisfied for $K$, then it is also satisfied for the ideal generated by $K_{d+1}$. Hence, if we assume that (b) and (c) are equivalent, we conclude that $K_{d+2}$ is lexsegment. Applying this argument repeatedly we see that all $K_{j}$ for $j \geq d$ are lexsegment. Thus we have shown (a). On the other hand, (a) trivially implies (b).

In order to prove the equivalence of (c) and (b) we first note that $\operatorname{Shad}\left(L^{f}(u)\right)$ is a lexsegment if and only if $\operatorname{Shad}\left(L^{f}(u)\right)=L^{f}\left(x_{1} u\right)$. In general one has $\operatorname{Shad}\left(L^{f}(u)\right) \subset$ $L^{f}\left(x_{1} u\right)$. If $x_{1}$ divides $u$, then by Lemma 2.1 , Shad $\left(L^{f}(u)\right)$ is a lexsegment, since $u / x_{1} \geq x_{n}^{d-1}$. In case $u=x_{2}^{d}$ and $w \in L^{f}\left(x_{1} u\right)$, then $w$ is in $\operatorname{Shad}\left(L^{f}(u)\right)$, since $w=x_{m(w)} w^{\prime \prime}$, with $w^{\prime \prime} \leq u$. Thus $\operatorname{Shad}\left(L^{f}(u)\right)=L^{f}\left(x_{1} u\right)$. This proves the implication $(\mathrm{c}) \Rightarrow(\mathrm{b})$.

Finally suppose that $u<x_{2}^{d}$. Then $x_{2}^{d+1} \in L^{f}\left(x_{1} u\right)$, but $x_{2}^{d+1}$ is not in $\operatorname{Shad}\left(L^{f}(u)\right)$. This proves the other implication and concludes the proof.

The next theorem, which is the main result of this section, treats arbitrary lexsegments which are not final. Hence, together with 2.2 , all completely lexsegment ideals are classified.

Theorem 2.3. Let $u=x_{1}^{a_{1}} \cdots x_{n}^{a_{n}}$ and $v=x_{1}^{b_{1}} \cdots x_{n}^{b_{n}}$ be monomials of degree $d$ in $R, v \neq x_{n}^{d}$, and let $I$ be the ideal generated by $L(u, v)$. Then $I$ is completely lexsegment if and only if $a_{1} \neq 0$, and one of the following conditions holds:

(a) $u=x_{1}^{p} x_{2}^{d-p}$ and $v=x_{1}^{p} x_{n}^{d-p}$ for some integer $p \leq d$;

(b) $a_{1} \neq b_{1}$, and for every $w<v$ there exists an index $i>1$ such that $x_{i} \mid w$ and $x_{1} w / x_{i} \leq u$. 
Proof. By Theorem 1.1, $I$ is completely lexsegment if and only if $\operatorname{Shad}(L(u, v))$ is a lexsegment, that is, if and only if $\operatorname{Shad}(L(u, v))=L\left(x_{1} u, x_{n} v\right)$.

If $a_{1}=0$, then $x_{2}^{d} \geq u$. Let $w \in M_{d}$ be such that $v>w$. Then $m=x_{1} w$ is in $L\left(x_{1} u, x_{n} v\right)$. Suppose that $m$ is in $\operatorname{Shad}(L(u, v))$; then $m=x_{j} n_{j}$ with $n_{j} \in L(u, v)$, for some $j$. But $n_{j} \leq u$ implies that $j=1$, and $n_{1}=w<v$, a contradiction. Thus $m \in L\left(x_{1} u, x_{n} v\right) \backslash \operatorname{Shad}(L(u, v))$.

Now let $0 \neq a_{1}=b_{1}=p$. Arguing as before, it is easy to see that if $u \neq$ $x_{1}^{p} x_{2}^{d-p}$, then $x_{1}^{p} x_{2}^{d-p+1} \in L\left(x_{1} u, x_{n} v\right) \backslash \operatorname{Shad}(L(u, v))$, and that if $v \neq x_{1}^{p} x_{n}^{d-p}$, then $x_{1}^{p+1} x_{n}^{d-p} \in L\left(x_{1} u, x_{n} v\right) \backslash \operatorname{Shad}(L(u, v))$.

Consider now the case $u=x_{1}^{p} x_{2}^{d-p}$ and $v=x_{1}^{p} x_{n}^{d-p}$. Let $m=x_{1}^{c_{1}} \cdots x_{n}^{c_{n}} \in$ $L\left(x_{1} u, x_{n} v\right)=L\left(x_{1}^{p+1} x_{2}^{d-p}, x_{1}^{p} x_{n}^{d-p+1}\right)$. One has $p+1 \geq c_{1} \geq p$. If $c_{1}=p+1$, then $m=x_{1} n$ with $n \in L(u, v)$, and if $c_{1}=p$, then $m=x_{M(m)} n$ with $n \in L(u, v)$. In both cases $m \in \operatorname{Shad}(L(u, v))$, and thus $L\left(x_{1} u, x_{n} v\right)=\operatorname{Shad}(L(u, v))$.

It remains to prove that for $a_{1} \neq 0$ and $a_{1} \neq b_{1}, I$ is completely lexsegment if and only if condition (b) holds. Let $J$ and $K$ be the ideals generated respectively by $L^{i}(v)$ and $L^{f}(u)$. It is known that $J$ is completely lexsegment, and since $a_{1} \neq 0$, $K$ is completely lexsegment, too; see 2.2 . In particular, $J \cap K$ is completely lexsegment. On the other hand, $I \subset J \cap K,(I)_{d}=(J \cap K)_{d}$, and by $1.3, J \cap K$ is generated in degree $d$ and $d+1$. Therefore it follows that $I$ is completely lexsegment if and only if $I=J \cap K$, that is, if and only if $J \cap K$ is generated in degree $d$. Let $L$ denote the ideal generated by all the monomials in $M_{d}$. The last condition is equivalent to the fact that

$$
\varphi: T_{2}(J) \oplus T_{2}(K) \rightarrow T_{2}(L)
$$

is surjective, cf. the proof of 1.3 .

For the further calculations we identify the Tor groups $T_{i}$ with the corresponding Koszul homology groups.

By $[\mathrm{AH}]$, the homology classes of the Koszul cycles $w^{\prime} e_{i} \wedge e_{M(w)}$ with $w \in M_{d}$, $i<M(w)$ form a basis of $T_{2}(L)$. We call cycles of this kind classical cycles. Analogously, homology classes of the same form, with $w \in L^{i}(v)$ form a basis of $T_{2}(J)$, and they are mapped by $\varphi$ to the corresponding homology classes. Thus all the elements $\left[w^{\prime} e_{i} \wedge e_{M(w)}\right]$ with $w \in L^{i}(v), i<M(w)$ are in the image of $\varphi$.

If $w \in L^{f}(u) \backslash L^{i}(v)$, that is $w<v$, then the differential of the corresponding classical cycle is

$$
d\left(w^{\prime} e_{i} \wedge e_{M(w)}\right)=w e_{i}-w^{\prime} x_{i} e_{M(w)} .
$$

If $i \neq 1$, then $w^{\prime} x_{i} \in K$; because if $w=x_{1}^{c_{1}} \cdots x_{n}^{c_{n}}$, then $c_{1} \leq b_{1}<a_{1}$, by hypothesis; thus $\left[w^{\prime} e_{i} \wedge e_{M(w)}\right] \in T_{2}(K)$. It follows that the cycles $w^{\prime} e_{i} \wedge e_{M(w)}$ with $w<v$ and $1 \neq i<M(w)$ are in the image of $\varphi$. Let $T$ be the $k$-subvector space of $T_{2}(L)$, generated by $\left[w^{\prime} e_{i} \wedge e_{M(w)}\right]$, for every $w \in M_{d}, 1 \neq i<M(w)$, and $\left[w^{\prime} e_{1} \wedge e_{M(w)}\right]$ for $w \in L^{i}(v)$. Then $\varphi$ is surjective if and only if the induced homomorphism,

$$
\bar{\varphi}: T_{2}(K) \rightarrow T_{2}(L) / T
$$

is surjective. Again applying $[\mathrm{AH}]$, we see that a basis of $T_{2}(K)$ is given by the homology classes of the cycles $z^{\prime \prime} e_{m(z)} \wedge e_{i}$ with $z \in L^{f}(u), i>m(z)$. We call such cycles non-classical cycles. It is clear that the image of $\bar{\varphi}$ is generated by the image of the non-classical cycles.

If $i \geq M(z)$, then $M\left(z^{\prime \prime} x_{i}\right)=i$, and $z^{\prime \prime} e_{m(z)} \wedge e_{i}=\left(z^{\prime \prime} x_{i}\right)^{\prime} e_{m(z)} \wedge e_{M\left(z^{\prime \prime} x_{i}\right)}$. Thus, if $m(z) \neq 1$, then $\varphi\left(\left[z^{\prime \prime} e_{m(z)} \wedge e_{i}\right]\right) \in T$, and hence $\bar{\varphi}\left(\left[z^{\prime \prime} e_{m(z)} \wedge e_{i}\right]\right)=0$. If 
$m(z)=1$, then $\bar{\varphi}\left(\left[z^{\prime \prime} e_{m(z)} \wedge e_{i}\right]\right)=\left[\left(z^{\prime \prime} x_{i}\right)^{\prime} e_{1} \wedge e_{M\left(z^{\prime \prime} x_{i}\right)}\right]$, which is one element of the canonical basis of $T_{2}(L) / T$.

Now let $i<M(z)$. Considering the boundary of $z^{\prime \prime} / x_{M(z)} e_{m(z)} \wedge e_{i} \wedge e_{M(z)}$, one gets

$$
\left[z^{\prime \prime} e_{m(z)} \wedge e_{i}\right]=-\left[z / x_{M(z)} e_{i} \wedge e_{M(z)}\right]+\left[z^{\prime \prime} x_{i} / x_{M(z)} e_{m(z)} \wedge e_{M(z)}\right] .
$$

Since $i \neq 1,\left[z / x_{M(z)} e_{i} \wedge e_{M(z)}\right]$ is in $T$. Moreover $M(z)=M\left(z^{\prime \prime} x_{i}\right)$, thus

$$
\bar{\varphi}\left(\left[z^{\prime \prime} e_{m(z)} \wedge e_{i}\right]\right)=\left[z^{\prime \prime} x_{i} / x_{M\left(z^{\prime \prime} x_{i}\right)} e_{m(z)} \wedge e_{M\left(z^{\prime \prime} x_{i}\right)}\right]=\left[\left(z^{\prime \prime} x_{i}\right)^{\prime} e_{m(z)} \wedge e_{M\left(z^{\prime \prime} x_{i}\right)}\right] .
$$

If $m(z) \neq 1$, it follows that $\bar{\varphi}\left(\left[z^{\prime \prime} e_{m(z)} \wedge e_{i}\right]\right)=0$.

If $m(z)=1$, then $\bar{\varphi}\left(\left[z^{\prime \prime} e_{m(z)} \wedge e_{i}\right]\right)=\left[\left(z^{\prime \prime} x_{i}\right)^{\prime} e_{1} \wedge e_{M\left(z^{\prime \prime} x_{i}\right)}\right]$ is an element of the canonical basis of $T_{2}(L) / T$. Summarizing our discussion, we see that a basis of the image of $\bar{\varphi}$ is given by the elements $\left[\left(z x_{i} / x_{1}\right)^{\prime} e_{1} \wedge e_{M\left(z x_{i} / x_{1}\right)}\right]$, with $z \in L^{f}(u)$, $i \neq 1$. Now we claim that $\bar{\varphi}$ is surjective if and only if condition (b) in the theorem holds, that is, if and only if for every $w<v$, there exist $1 \leq i \leq n$ and $z \in L^{f}(u)$ such that $w=z x_{i} / x_{1}$. In fact, the canonical basis of $T_{2}(L) / T$ is given by the cycles $\left[w^{\prime} e_{1} \wedge e_{M(w)}\right]$ with $w<v$. It is clear that if (b) holds, then $\left[w^{\prime} e_{1} \wedge e_{M(w)}\right]=$ $\left[\left(z x_{i} / x_{1}\right)^{\prime} e_{1} \wedge e_{M\left(z x_{i} / x_{1}\right)}\right]$ is in the image of $\bar{\varphi}$, thus $\operatorname{Im} \bar{\varphi}=T_{2}(L) / T$. On the other hand, if $\bar{\varphi}$ is surjective, then the restriction to every multigraded component is surjective. Since every element $\left[w^{\prime} e_{1} \wedge e_{M(w)}\right]$ of the canonical basis of $T_{2}(L) / T$ has a different multidegree, it must be the image of a certain non-classical cycle in $T_{2}(K)$. Thus condition (b) holds.

Remark 2.4. The sufficient condition given by Hulett and Martin (Lemma 2.1) is not necessary. Consider for example the lexsegment given by $u=x_{1}^{2} x_{3}$ and $v=x_{1} x_{2} x_{3}$. Then $u / x_{1}<v / x_{3}$, but $L(u, v)$ is completely lexsegment.

\section{LEXSEGMENT IDEALS WITH LINEAR RESOLUTION}

This section is devoted to the proof of the following result.

Theorem 3.1. Let I be a lexsegment ideal. With the notation of 2.3 the ideal I has a linear resolution in the following cases:

(1) $a_{1} \neq 0$, and I satisfies $2.3(a)$;

(2) $0 \neq a_{1} \neq b_{1}$, and $x_{1} w / x_{M(w)} \leq u$ for every $w<v$;

(3) $I$ is an initial or final lexsegment ideal.

Remark 3.2. (a) In the cases (1) and (2) I is completely lexsegment. However not all completely lexsegment ideals have a linear resolution. The following example is due to Aramova: The ideal $I=\left(L\left(a b d, b^{2} c\right)\right)$ in $k[a, b, c, d]$ is completely lexsegment, but has no linear resolution.

(b) In their paper [HM] Hulett and Martin also give a sufficient condition for lexsegment ideals to have a linear resolution. Our condition (2) is implied by the condition of Hulett and Martin, but the converse is not true, as can be shown by the example in Remark 2.4.

Proof. Let $u \geq v$ be two monomials of degree $d, u=x_{1}^{a_{1}} \cdots x_{n}^{a_{n}}$ and $v=x_{1}^{b_{1}} \cdots x_{n}^{b_{n}}$, and let $I$ be the ideal generated by $L(u, v)$. Initial and final lexsegment ideals are both stable and generated in a fixed degree. Thus it follows from [EK] that these have a linear resolution.

We may now assume that $u \neq x_{1}^{d}$ and $v \neq x_{n}^{d}$. 
In case $(1), I=\left(L\left(x_{1}^{p} x_{2}^{d-p}, x_{1}^{p} x_{n}^{d-p}\right)\right)$, for some $0<p<d$. It is easy to see that $I$ is isomorphic, as an $R$-module, to the final lexsegment ideal generated by $L\left(x_{2}^{d-p}, x_{n}^{d-p}\right)=L^{f}\left(x_{2}^{d-p}\right)$, and hence it has a linear resolution.

Suppose now that condition (2) holds. In order to prove that $I$ has a linear resolution it is enough to show that

$$
\varphi: T_{s+1}(J) \oplus T_{s+1}(K) \rightarrow T_{s+1}(L)
$$

is surjective for every $s \geq 2$, where $L$ is the ideal generated by all monomials of degree $d$. By $[\mathrm{AH}]$, the homology classes of the cycles $w^{\prime} e_{i_{1}} \wedge \cdots \wedge e_{i_{s}} \wedge e_{M(w)}$, with $w \in M_{d}$ and $1 \leq i_{1}<i_{2}<\cdots<i_{s}<M(w)$ form a basis of $T_{s+1}(L)$. It is clear that if $w$ is in $L^{i}(v)$, then $\left[w^{\prime} e_{i_{1}} \wedge \cdots \wedge e_{i_{s}} \wedge e_{M(w)}\right]$ is in the image of $\varphi$.

Note that

$$
\begin{aligned}
& d\left(w^{\prime} e_{i_{1}} \wedge \cdots \wedge e_{i_{s}} \wedge e_{M(w)}\right) \\
& \quad=w e_{i_{1}} \wedge \cdots \wedge e_{i_{s}}+\sum_{j=1}^{s} \pm w^{\prime} x_{i_{j}} e_{i_{1}} \wedge \cdots \wedge e_{i_{j-1}} \wedge e_{i_{j+1}} \wedge \cdots \wedge e_{i_{s}} \wedge e_{M(w)} .
\end{aligned}
$$

Therefore, if $w \in L^{f}(u) \backslash L^{i}(v)$, and $i_{1} \neq 1$, then $w^{\prime} x_{i_{j}} \in L^{f}(u)$ for every $i_{j}$, and so $w^{\prime} e_{i_{1}} \wedge \cdots \wedge e_{i_{s}} \wedge e_{M(w)}$ is a cycle whose homology class belongs to $T_{s+1}(K)$. Thus $\left[w^{\prime} e_{i_{1}} \wedge \cdots \wedge e_{i_{s}} \wedge e_{M(w)}\right]$ is in the image of $\varphi$.

Hence in order to prove that $\varphi$ is surjective it remains to show that the elements of the form $\left[w^{\prime} e_{1} \wedge e_{i_{2}} \wedge \cdots \wedge e_{i_{s}} \wedge e_{M(w)}\right]$ are in the image of $\varphi$, for every $w<v$. By hypothesis there exists $z \in L^{f}(u)$ such that $w=z x_{M(w)} / x_{1}$. Since $z \neq x_{1}^{d}$ it follows that $M(z) \leq M(w)$. Therefore, $w^{\prime}=\left(z x_{M(w)} / x_{1}\right)^{\prime}=z / x_{1}=z^{\prime \prime}$ and so $w^{\prime} e_{1} \wedge e_{i_{2}} \wedge \cdots \wedge e_{i_{s}} \wedge e_{M(w)}=z^{\prime \prime} e_{m(z)} \wedge e_{i_{2}} \wedge \cdots \wedge e_{i_{s}} \wedge e_{M(w)}$ is a non-classical cycle. Hence $\left[w^{\prime} e_{1} \wedge e_{i_{2}} \wedge \cdots \wedge e_{i_{s}} \wedge e_{M(w)}\right] \in \operatorname{Im} \varphi$, as desired.

\section{REFERENCES}

[AH] A. Aramova and J. Herzog, Koszul cycles and Eliahou-Kervaire type resolutions, J. Algebra 183 (1996), 347 - 370. MR 97c:13009

[De] T. Deery, Rev-Lex segment ideals and minimal Betti numbers, preprint, 1996.

[D] E. De Negri, Toric rings generated by stable sets of monomials, preprint 1996.

[EK] S. Eliahou and M. Kervaire, Minimal resolutions of some monomial ideals, J. Algebra 129 (1990), 1 - 25. MR 91b:13019

[HM] H. A. Hulett and H. M. Martin, Betti numbers of lexsegment ideals, preprint 1996.

FB 6 Mathematik und Informatik, Universität-GHS-Essen, Postfach 103764, Essen 45117, GERMANY

E-mail address, Emanuela De Negri: mat304@uni-essen.de

E-mail address, Jürgen Herzog: mat300@uni-essen.de 